\title{
Dark Matter Constituents
}

\author{
L. Bergström ${ }^{\mathrm{a} *}$ \\ a Physics Department, Stockholm University, \\ AlbaNova University Centre, S-106 91 Stockholm, Sweden
}

As cosmology has entered a phase of precision experiments, the content of the universe has been established to contain interesting and not yet fully understood components, namely dark energy and dark matter. While the cause and exact nature of the dark energy remains mysterious, there is greater hope to connect the dark matter to current models of particle physics. Supersymmetric models provide several excellent candidates for dark matter, with the lightest neutralino the prime example. This and other dark matter candidates are discussed, and prospects for their detection summarized. Some methods of detection are explained, and indications of signals in present data are critically examined.

\section{INTRODUCTION}

The question of the nature of the average composition of the Universe is getting more interesting than ever. As new observations have arrived, the picture emerging has become quite puzzling, with the favoured model now containing both dark energy (vacuum energy, "cosmological constant" or perhaps a slowly evolving scalar field, "quintessence") and dark, non-baryonic matter.

The recent supernova discovery of acceleration of the cosmic expansion [1 taken in combination with the spectacular new WMAP microwave background measurements 2] has led to a "concordande model" $(\Lambda \mathrm{CDM})$ :

$\left\{\begin{array}{l}\Omega_{M}=0.3 \\ \Omega_{\Lambda}=0.7\end{array}\right.$

This is in nice agreement with the inflationary prediction of the matter and cosmological constant contributions adding up to unity, $\Omega_{t o t}=1$, but produces the mystery of having a non-zero but exceedingly small value of the cosmological constant $\Lambda$ (compared to the natural scale of gravity, which is the Planck scale). However, the supernova data (of two independent groups) and CMBR measurement in combination with the measured range of the Hubble constant $\left(H_{0} \simeq 70\right.$ $\left.\mathrm{km} / \mathrm{s} \mathrm{Mpc}^{-1}, h \equiv H_{0} / 100=0.7 \pm 0.1\right)$ has more or less forced this model upon us.

\footnotetext{
*Supported by the Swedish Research Council (VR)
}

The WMAP data and Big Bang nucleosynthesis $(\mathrm{BBN})$ deuterium data 3 , agree on the baryonic contribution $\Omega_{b}$ to $\Omega_{M}$ :

$\Omega_{b} h^{2} \simeq 0.023 \pm 0.002$.

This means that non-baryonic dark matter is required beyond any doubt, since $h=0.7 \pm 0.1$ gives the baryonic contribution $\Omega_{b}$ less than around 0.08 , so much less than the measured $\Omega_{M}$.

Weakly interacting massive particle candidates (WIMPs) for dark matter are generically found to decouple at a temperature that is roughly $m_{\text {WIMP }} / 20$, which means that they are nonrelativistic and behave as CDM when structure forms.

WIMPs have the advantage that their relic density is determined by the calculable departure from thermal equilibrium in the early universe, and give without difficulties a contribution to $\Omega$ which may be close to the observed value.

\section{DARK MATTER CANDIDATES}

\subsection{Neutrinos}

With the masses of neutrinos in view of observed oscillations being definitely non-zero, we may for the first time say with certainty that non-baryonic dark matter exists. However, the neutrino contribution to $\Omega_{M}$ is most likely very small. For small mass neutrinos (hot dark matter, 
HDM), the relic abundance is given by

$\Omega_{\nu} h^{2}=\frac{\sum_{i} m_{\nu_{i}}}{94 \mathrm{eV}}$.

For the kind of mass values indicated by the Super-Kamiokande measurements, $0.01-0.1 \mathrm{eV}$ (unless there are unnatural neutrino mass degeneracies), we see that although neutrinos indeed contribute to non-baryonic dark matter, they do so at the level or $10^{-2}$ or less. This may seem tiny from the cosmological point of view, but is still about as much as all visible material like stars, gas, dust, etc contribute to the energy density of the Universe.

The WMAP results, especially when augmented by large galaxy survey data such as SDSS [4] or 2DF [5], also give very little room for the standard neutrino contribution to dark matter. Since the distribution of matter is quite different in CDM and HDM models (with the latter lacking small-scale power due to free-streaming), one may use the observed distribution to put a direct upper bound to the sum of all neutrino masses which turns out to be of the order of an $\mathrm{eV}$ or so (the WMAP limit is $0.23 \mathrm{eV}$ for all three neutrinos degenerate, but this includes less understood smaller scale data from the Lyman alpha forest; for a discussion see also [6]). The advantage of the cosmological limit is that it is sensitive to the absolute mass scale of neutrinos whereas neutrino oscillations only probe mass differences.

In principle, one could imagine having a sterile neutrino as dark matter, if it is non-thermally produced, e.g., generated through mixing with the active neutrinos. Generally, this candidate will have to have a mass in the $\mathrm{keV}$ to $\mathrm{MeV}$ range and would act as something inbetween cold and hot dark matter (sometimes named "warm dark matter", WDM). An unpleasant feature of these models is a necessary, delicate finetuning of the mixing angle versus mass to get the right abundance, but models of this kind have been constructed which may perhaps evade experimental constraints [78.

\subsection{Other Solutions to the Dark Matter Problem}

A class of dark matter candidates, which should be kept in mind, are super-heavy relics which were produced by some non-thermal mechanism in the early Universe. Examples are given by "wimpzillas" 9].

At the other end of the mass spectrum are axions, light pseudoscalar mesons that appear in all string theories and which are needed to explain the weakness of $\mathrm{CP}$ violation in strong interactions. There are experiments which search for these particles in mass ranges interesting for cosmology [10.

There has recently been interest in dark matter in the form of topological, extended objects, "Q-balls" which may or may not be supersymmetric and may or may not be stable on cosmological time scales (see 11] for a recent review). In certain scenarios, supersymmetric Q-balls may decay into the lightest neutralino, giving a similar scenario as in the MSSM, but with different phenomenology. For instance, the usual bounds on the relic density for thermally produced neutralinos may be evaded.

\section{SUPERSYMMETRIC DARK MAT- TER}

One of the prime candidates for the nonbaryonic component is provided by the lightest supersymmetric particle, plausibly the lightest neutralino $\chi$.

The lightest neutralino $\chi$ is a mixture of the supersymmetric partners of the photon, the $Z$ and the neutral part of the two Higgs doublets present in the minimal extension of the supersymmetric standard model (for reviews see, e.g., 1213). The attractiveness of this dark matter candidate stems from the fact that its generic couplings and mass range naturally gives a relic density in the required range to explain halo dark matter. Besides, its motivation from particle physics, which was originally based on solving the so-called hierarchy problem (the puzzling discrepancy between the mass scales of electroweak interactions and gravity), has become stronger due to the apparent need for $100 \mathrm{GeV}-10 \mathrm{TeV}$ scale supersymmetry to achieve unification of the gauge couplings in view of LEP results 14, and the prediction that the lightest Higgs boson should be below 135 $\mathrm{GeV}$, as may be favoured by LEP data [15]. 
Recently, there has been discussion about the constraints on the MSSM which follow from the measurements of $(g-2)_{\mu}$, the anomalous magnetic moment of the muon [16. The new set of data recently released shows a mild discrepancy with the Standard Model (but only at the $2-3 \sigma$ level), which could in principle be due to supersymmetry [17.

The relic density calculation in the MSSM for a given set of parameters is nowadays accurate a few $\%$ or so, thus matching the precision given by cosmological measurements. A recent important improvement is the inclusion of coannihilations, which can change the relic abundance by a large factor in some instances [18. Much of the effort that has gone into this field has resulted in publicly available computer program packages, for instance DarkSUSY [19].

\subsection{Detection methods \\ 3.1.1. Direct detection}

If neutralinos are indeed the CDM needed on galaxy scales and larger, there should be a substantial flux of these particles in the Milky Way halo. Since the interaction strength is essentially given by the same weak couplings as, e.g., for neutrinos there is a non-negligible chance of detecting them in low-background counting experiments. Due to the large parameter space of MSSM, even with the simplifying assumptions above, there is a rather wide span of predictions for the event rate in detectors of various types.

The experimental situation is becoming interesting as several direct detection experiments after many years of continuing sophistication are starting to probe interesting parts of the parameter space of the MSSM, given reasonable, central values of the astrophysical and nuclear physics parameters.

The most enigmatic finding is that of DAMA 20, which after seven years of running in the lowbackground environment in Gran Sasso, now reports a visible seasonal variation 21] of the scattering rate which is formally at the $6 \sigma$ level.

This seems to be difficult to reconcile with results from the CDMS 22] and EDELWEISS experiments [23], which have recently improved their limits by an order of magnitude. The data released essentially exclude all the parameter range for supersymmetric dark matter indicated by the DAMA experiment 20]. However, it is difficult to compare the experiments directly, since they use different materials and very different techniques. For instance, it is not excluded that a large spin-dependent interaction may explain the DAMA results 24.

Although neutralino dark matter may, with careful choice of physical and astrophysical parameters 25, explain the DAMA results, the rates in minimal supersymmetry are generally lower. Even the CDMS and EDELWEISS experiments are so far only scratching the surface of the parameter space that is provided by the MSSM. However, a new generation of experiments is being prepared with an order of magnitude better sensitivity [26]. The urgent challenge to the experimental dark matter community is now to build an independent, and preferably more sensitive detector which is similar enough to DAMA to enable a direct comparison [27].

\subsection{Indirect detection}

There is also the possibility of indirect detection through neutralino annihilation in the galactic halo or in astrophysical objects like the Sun and the Earth. This is becoming a promising method thanks to very powerful new detectors for cosmic gamma rays and neutrinos planned and under construction.

There has recently been balloon-borne detection experiments 28], where an excess of positrons over that expected from ordinary sources has been found. However, since there are many other possibilities to create positrons by astrophysical sources, e.g. near the centre of the Milky Way, the interpretation is not yet conclusive. In particular, it has been difficult to reproduce the apparent "bump" seen at $7-8 \mathrm{GeV}$ in any model based on WIMP annihilation (for recent attempts, see [29]).

Antiprotons, $\bar{p}$, from neutralino annihilations were long hoped to give a useful signal [30, and there have been several balloon-borne experiments 3132 performed and a very ambitious space experiment, AMS, to search for antimatter is under way 33. For kinematical reasons, 
antiprotons created by pair-production in cosmic ray collisions with interstellar gas and dust are born with relatively high energy, whereas antiprotons from neutralino annihilation populate also the sub-100 MeV energy band.

However, it was found 3435] that the cosmicray induced antiprotons may populate also the low-energy region to a greater extent than previously thought, making the extraction of an eventual supersymmetric signal much more difficult. Recently, a thorough reanalysis [36 has arrived at the same conclusion.

Another problem that plagues estimates of the signal strength of both positrons and antiprotons is the uncertainty of the galactic propagation model and solar wind modulation.

Even allowing for large such systematic effects, the measured antiproton flux gives, however, rather stringent limits on MSSM models with the highest annihilation rates. One can also use the experimental upper limits to bound from below the lifetime of hypothetical $R$-parity violating decaying neutralinos 37. There may in some scenarios with a clumpy halo 38 (which enhances the annihilation rate) be a possibility to detect heavy neutralinos through spectral features above several $\mathrm{GeV}$ 39, something which will be probed by the upcoming PAMELA satellite project [40.

A very rare process in proton-proton collisions, antideuteron production, may be less rare in neutralino annihilation [41. However, the fluxes are so small that the possibility of detection seems marginal even in the AMS experiment.

With these problems of positrons and antiprotons, one would expect that problems of gamma rays and neutrinos are similar, if they arise from secondary decays in the annihilation process in the halo. For instance, the gamma ray spectrum arising from the fragmentation of fermion and gauge boson final states is quite featureless and gives the bulk of the gammas at low energy where the cosmic gamma ray background is severe. Also, the density of neutralinos in the halo is not large enough to give a measurable flux of secondary neutrinos, unless the dark matter halo is very clumpy [38. However, neutrinos can escape from the centre of the Sun or Earth, where neutralinos may have been gravitationally trapped and therefore their density enhanced.

Also, gamma-rays may be emitted from places where the dark matter density is substantially higher than average, e.g. in dark matter clumps [38, or near the galactic center [42]. For a recent attempt to interpret the various anomalies in the measured fluxes of gamma rays, positrons and antiprotons in terms of neutralino dark matter, see 43. As a general comment, one should observe that the systematic uncertainties that come from unknown astrophysical parameters and propagation functions are much larger than the statistical errors used in 43 .

\subsection{Gamma ray lines}

Gamma rays may also result from loop-induced annihilations [4445] $\chi \chi \rightarrow \gamma \gamma$ or $\chi \chi \rightarrow Z \gamma$.

This would give monoenergetic photons with $E_{\gamma}=m_{\chi}$ or $E_{\gamma}=m_{\chi}\left(1-m_{Z}^{2} / 4 m_{\chi}^{2}\right)$ from the halo. The detection probability of a gamma line signal depends on the very poorly known density profile of the dark matter halo. N-body simulations have given a clue to the final halo profile obtained by hierarchical clustering in a CDM scenario [4]. It turns out that the universal halo profile found in these simulations has a rather significant enhancement $\propto 1 / r$ near the halo centre. If applicable to the Milky Way, this would lead to a much enhanced annihilation rate towards the galactic centre, and also to a very characteristic angular dependence of the line (and continuous gamma) signal. This would be very beneficial when discriminating against the galactic and extragalactic $\gamma$ ray background, and Air Cherenkov Telescopes (ACTs) would be eminently suited to look for these signals, if the energy resolution is at the $10-20 \%$ level.

The models which give the highest rates should be within reach of the new generation of ACTs presently being constructed. These will have an effective area of almost $10^{5} \mathrm{~m}^{2}$, a threshold of some tens of $\mathrm{GeV}$ and an energy resolution approaching $10 \%$. For low-mass models, the spaceborne telescope GLAST will have a better sensitivity. (See [4] for details.)

Another possibility to detect dark matter in gamma-rays has recently been investigated [48]. 
If N-body simulations of structure formation are taken seriously, it appears that the average enhancement of the integrated signal from all cosmic structure in the universe would be several orders of magnitude compared to the case when the dark matter density only scales with the cosmic dilution factor $(1+z)^{3}$. The signature would be a continuum from neutralino annihilations plus a characteristic redshift-smeared line with a very rapid fall-off beyond the energy corresponding to the neutralino mass.

\subsection{Indirect detection through neutrinos}

More model-independent predictions, where essentially only the relatively well-determined local halo dark matter density is of importance, can be made for neutrinos from the centre of the Sun or Earth, where neutralinos may have been gravitationally trapped and therefore their density enhanced. (However, also the less well known velocity distribution also does affect the capture rate, weakening the predictions somewhat, especially for the Earth.) As neutralinos annihilate, many of the possible final states give after decays and perhaps hadronization energetic neutrinos which will propagate out from the interior of the Sun or Earth. In particular, the muon neutrinos are useful for indirect detection of neutralino annihilation processes, since muons have a quite long range in a suitable detector medium like ice or water. Therefore they can be detected through their Cherenkov radiation after having been produced at or near the detector, through the action of a charged current weak interaction $\nu_{\mu}+A \rightarrow \mu+X$.

Detection of neutralino annihilation into neutrinos is one of the most promising indirect detection methods, and will be subject to extensive experimental investigations in view of the new neutrino telescopes (AMANDA/IceCUBE, Baikal, NESTOR, ANTARES) planned or under construction 49. The advantage shared with gamma rays is that neutrinos keep their original direction.

The detector threshold energy, for "small" neutrino telescopes like Baksan, MACRO (now discontinued) and Super-Kamiokande is around 1 $\mathrm{GeV}$. Large area neutrino telescopes in the ocean or in Antarctic ice typically have thresholds of the order of tens of $\mathrm{GeV}$, which makes them sensitive mainly to heavy neutralinos (above $100 \mathrm{GeV}$ ) 50 .

The detector response is weighted towards high neutrino energies, both because the cross section $\sigma_{\nu}$ rises approximately linearly with energy and because the average muon energy, and therefore the range, also grow approximately linearly with $E_{\nu}$. Therefore, final states which give a hard neutrino spectrum (such as heavy quarks, $\tau$ leptons and $W$ or $Z$ bosons) are usually more important than the soft spectrum arising from light quarks and gluons.

The capture rate, and therefore the signal rate, of the Earth is very similar to the scattering rate in the materials used for spin-independent detection. It seems therefore that the new generation of direct detection experiments generally have a greater sensitivity than the new neutrino telescopes searching for signals from the Earth [51. For the Sun, however, neutrinos provide a complementary and in some cases superior method [13.

\section{NON-SUSY CANDIDATES}

The phenomenology of supersymmetric dark matter (neutralinos) may be very similar for other types of weakly interacting massive particles (WIMPs). However, one can also imagine models where the WIMP only couples to leptons. These leptonic WIMPs, or LIMPs, may at first seem essentially undetectable in present-day experiments. It may be shown, however, that in most cases they necessarily give energetic gamma rays in their annihilations, due to higher-order processes 52 .

Of course, the current interest among theoretical physicists in exotic, but at least partly realistic models like branes and large extra dimensions has also led to a number of dark matter models. Just as an example, models where the Standard Model fields live in compactified, "universal" extra dimension has a rich dark matter phenomenology, where an orbifold parity plays the role of R-parity in supersymmetric models. Detection rates turn out to be similar to the SUSY case but some interesting differences in the detailed phenomenology 
53.

It finally should be noted with caution, that there are many models for dark matter where the candidate only interacts very weakly, e.g. gravitationally, with other matter. For some models, there still may exist cosmological signals from early decays [54, but it may not be excluded that detection by other methods is impossible. Still one may hope to learn more about the detailed distribution of dark matter, for instance by studying their effects on gravitational lensing. There are in fact studies 55 that interpret flux ratios of multiple gravitational images in terms of clumpiness of galactic halos, in rough agreement with what is found in N-body simulations.

\section{CONCLUSIONS}

To conclude, non-baryonic cold dark matter is required to explain new cosmological data, in particular on the microwave background. The fact that the favoured value of $\Omega_{M}$ has gone down during the last decade from near 1 to around 0.3 is good news for detection, since larger cross sections generally means lower relic density. In particular this is true for the main particle physics candidate, the neutralino, which we have presented in some detail here. Direct detection experiments are rapidly developing their discovery potential. Indirect detection methods may be very useful complements. In particular, new gamma-ray and neutrino telescopes may have the sensitivity to rule out or confirm the supersymmetry solution of the dark matter problem. And of course, when the LHC starts taking data, we may get direct clues to the nature of the particle that most likely makes up the dark matter.

\section{ACKNOWLEDGEMENTS}

I wish to thank my collaborators, in particular Ted Baltz, Joakim Edsjö, Paolo Gondolo, Mia Schelke and Piero Ullio, for many helpful discussions. This work has been supported in part by the Swedish Research Council (VR).

\section{REFERENCES}

1. S. Perlmutter et al., Astrophys. J. 483565 (1997); S. Perlmutter et al., Astrophys. J. 517, 565 (1999); P.M. Garnavich et al., Astrophys. J. Lett. 493 (1998) 53; A. Riess et al., Astron. J. 116, 1009 (1998); J. L. Tonry et al., Astrophys. J. 594, 1 (2003); R. A. Knop et al., arXiv:astro-ph/0309368

2. C. L. Bennett et al., Astrophys. J. Suppl. 148, 1 (2003).

3. D. Tytler, J. M. O'Meara, N. Suzuki and D. Lubin, Physica Scripta T85, 12 (2000).

4. M. Tegmark et al. [SDSS Collaboration], arXiv:astro-ph/0310723

5. O. Elgaroy et al., Phys. Rev. Lett. 89, 061301 (2002).

6. S. Hannestad, JCAP 0305, 004 (2003).

7. A. D. Dolgov and S. H. Hansen, Astropart. Phys. 16, 339 (2002).

8. K. N. Abazajian and G. M. Fuller, Phys. Rev. D 66, 023526 (2002).

9. D. J. H. Chung, E. W. Kolb and A. Riotto, Phys. Rev. D 59, 023501 (1999).

10. S. J. Asztalos et al., Astrophys. J. 571, L27 (2002); M. Tada et al., Nucl. Phys. Proc. Suppl. 72, 164 (1999).

11. K. Enqvist and A. Mazumdar, Phys. Rep. 380, 99 (2003).

12. G. Jungman, M. Kamionkowski and K. Griest, Phys. Rep. 267 (1996) 195.

13. L. Bergström, Rep. Prog. Phys. 63, 793 (2000).

14. U. Amaldi, W. de Boer and H. Furstenau, Phys. Lett. B260 (1991) 447.

15. R. Barate et al., Phys. Lett. B 565, 61 (2003).

16. G. W. Bennett et al. [Muon g-2 Collaboration], Phys. Rev. Lett. 89, 101804 (2002) [Erratum-ibid. 89, 129903 (2002)].

17. E. A. Baltz and P. Gondolo, Phys. Rev. D 67, 063503 (2003); Y. G. Kim, T. Nihei, L. Roszkowski and R. Ruiz de Austri, JHEP 0212, 034 (2002).

18. J. R. Ellis, T. Falk, K. A. Olive and M. Srednicki, Astropart. Phys. 13, (2000) 181 [Erratum-ibid. 15, (2001) 413]; M. E. Gomez, G. Lazarides and C. Pallis, Phys. Lett. B 487, 313 (2000); 
T. Nihei, L. Roszkowski and R. Ruiz de Austri, JHEP 0207, 024 (2002);

H. Baer, C. Balazs and A. Belyaev, JHEP 0203, 042 (2002);

J. Edsjö, M. Schelke, P. Ullio and P. Gondolo, JCAP 0304, 001 (2003).

19. P. Gondolo, J. Edsjö, L. Bergström, P. Ullio and E. A. Baltz, arXiv:astro-ph/0012234

20. R. Bernabei et al., Riv. Nuovo Cim. 26, 1 (2003).

21. A. K. Drukier, K. Freese and D. N. Spergel, Phys. Rev. D 33, 3495 (1986); K. Freese, J. A. Frieman and A. Gould, Phys. Rev. D 37, 3388 (1988).

22. D. S. Akerib et al. [CDMS Collaboration], Phys. Rev. D 68, 082002 (2003).

23. A. Benoit et al., Phys. Lett. B545, (2002) 43.

24. A. Kurylov and M. Kamionkowski, arXiv:hep-ph/0307185

25. A. Bottino, F. Donato, N. Fornengo and S. Scopel, Phys. Rev. D 63, 125003 (2001).

26. A. Morales, these proceedings; W. Seidel, these Proceedings.

27. See J. Bahcall, concluding remarks, these Proceedings.

28. M. A. DuVernois et al., Astrophys. J. 559, 296 (2001).

29. E. A. Baltz, J. Edsjö, K. Freese and P. Gondolo, Phys. Rev. D 65, 063511 (2002); G. L. Kane, L. T. Wang and J. D. Wells, Phys. Rev. D 65, 057701 (2002).

30. G. Jungman and M.Kamionkowski, Phys. Rev. D49, 2316 (1994); A. Bottino, C. Favero, N. Fornengo, G. Mignola, Astropart. Phys. 3, 77 (1995).

31. CAPRICE Collaboration, M. Boezio et al., Astrphys. J. 487, 415 (1997).

32. BESS Collaboration, A. Moiseev et al., Astrophys. J. 474, 479 (1997).

33. S.P. Ahlen et al., Nucl. Instr. Methods A350, 351 (1994); J. Alcaraz et al., Phys. Lett. B461, 387 (1999).

34. L. Bergström, J. Edsjö and P. Ullio, Astrophys. J. 526, 215 (1999).

35. J.W. Bieber et al., Phys. Rev. Lett. 83, 674 (1999).

36. F. Donato, N. Fornengo, D. Maurin, P. Salati and R. Taillet, arXiv:astro-ph/0306312
37. E.A. Baltz and P. Gondolo, Phys. Rev. D57, 2969 (1998).

38. L. Bergström, J. Edsjö, P. Gondolo and P. Ullio, Phys. Rev. D59, 043506 (1999); C. Calcaneo-Roldan and B. Moore, Phys. Rev. D 62, 123005 (2000); A. Tasitsiomi and A. V. Olinto, Phys. Rev. D 66, 083006 (2002).

39. P. Ullio, astro-ph/9904086 (1999).

40. O. Adriani et al., Nucl. Instrum. Meth. A 478, 114 (2002).

41. F. Donato, N. Fornengo and P. Salati, Phys. Rev. D 62, 043003 (2000).

42. G. Bertone, G. Sigl and J. Silk, Mon. Not. Roy. Astron. Soc. 326, 799 (2001).

43. W. de Boer, M. Herold, C. Sander and V. Zhukov, arXiv:hep-ph/0309029

44. L. Bergström and H. Snellman, Phys. Rev. D37, 3737 (1988); A. Bouquet, P. Salati and J. Silk, Phys. Rev. D40, 3168 (1989); G. Jungman and M. Kamionkowski, Phys. Rev. D51, 3121 (1995).

45. L. Bergström and J. Kaplan, Astropart, Phys. 2, 261 (1994). P. Ullio and L. Bergström, Phys. Rev. D 57, 1962 (1998).

46. J.F. Navarro, C.S. Frenk and S.D.M. White, Astrophys. J. 462, 563 (1996).

47. L. Bergström, P. Ullio and J. H. Buckley, Astropart. Phys. 9, 137 (1998).

48. P. Ullio, L. Bergström, J. Edsjö and C. Lacey, Phys. Rev. D 66, 123502 (2002).

49. T.K. Gaisser, F. Halzen and T. Stanev, Phys. Rep. 258, 173 (1995).

50. L. Bergström, J. Edsjö and P. Gondolo, Phys. Rev. D58, 103519 (1998).

51. M. Kamionkowski, G. Jungman, K. Griest and B. Sadoulet, Phys. Rev. Lett. 74, 5174 (1995).

52. E. A. Baltz and L. Bergström, Phys. Rev. D 67, 043516 (2003).

53. H.-C. Cheng, J.L. Feng and K.T. Matchev, Phys. Rev. Lett. 89, 211301 (2002); G. Servant and T.M.P. Tait, New J. Phys. 4, 99 (2000); D. Hooper and G.D. Kribs, Phys. Rev. D67, 055003 (2003).

54. J. L. Feng, A. Rajaraman and F. Takayama, Phys. Rev. D 68, 063504 (2003).

55. N. Dalal and C.S. Kochanek, Astrophys. J. 572, 25 (2002). 